\title{
Growth Scale Optimization of Discrete Innovation Population Systems with Multichoice Goal Programming
}

\author{
Su-Lan Zhai, ${ }^{1}$ Ying Liu, ${ }^{2}$ Sheng-Yuan Wang ${ }^{D},{ }^{2}$ and Xiao-Lan $\mathrm{Wu}^{2}$ \\ ${ }^{1}$ Department of Immunology, Nanjing Medical University, Nanjing, Jiangsu, China \\ ${ }^{2}$ Nanjing Xiaozhuang University, Nanjing, Jiangsu, China \\ Correspondence should be addressed to Sheng-Yuan Wang; 56439976@qq.com
}

Received 16 June 2021; Revised 14 September 2021; Accepted 15 September 2021; Published 4 October 2021

Academic Editor: Baogui Xin

Copyright (c) 2021 Su-Lan Zhai et al. This is an open access article distributed under the Creative Commons Attribution License, which permits unrestricted use, distribution, and reproduction in any medium, provided the original work is properly cited.

How are limited resources efficiently allocated among different innovation populations? The performances of different innovation populations are quite different with either synergy or competition between them. If the innovation population is kept under an appropriate scale, full use can be made of the allocated resources. The maximization of the development and performance for a certain scale of innovation population is a typical multichoice development problem. Therefore, the scale optimization of the innovation population should be analyzed. According to the population dynamics, a resource constraint model for the growth of innovation population is developed, and the growth of innovation population under resource constraints is in equilibrium accordingly. With the help of a multichoice goal programming model, the scale optimization of innovation population performance can be obtained. The results of the resource constraint model and multichoice goal programming model are used to determine the optimal scale of the innovation population. From the panel data of the innovation population in Jiangsu Province from 2000 to 2017, we have found that R\&D investment was the main innovation resource variable and that patent number was the main innovation output variable. Based on these data, the scale optimization of the innovation population under resource constraints can be calculated. The results of the study show that, in the observation period, the enterprise innovation population is often in the appropriate scale state. The scale development of enterprise innovation population is often more suitable for innovation ecosystem than that of scientific research institutions. According to these results, the government can provide appropriate guiding policies and incentives for different innovation populations. The innovative population can adjust its own development strategy and plan in time accordingly.

\section{Introduction}

In real economic activities, the essence of enterprises' innovation behavior is to seek differences. The competitive advantages and the improvement of production efficiency brought by enterprises through innovation are the roots of realizing economic growth and sustainable change. Since endogenous growth theory regards technological progress as the source of economic growth, the research on technological innovation and economic growth is currently a hot topic in academic circles. However, the objective hypothesis of "innovation homogeneity" is always considered a defect of endogenous growth theory [1]. In fact, the differences in production technology, geographical environment, corporate culture, and factor accumulation in real life often led to the obvious heterogeneity of different enterprises.

Innovation activity is a systematic engineering issue. It is difficult for a single organization to have all the resources they need for innovation. The construction of an innovation ecosystem, an open, nonlinear, multilevel, and complex system with dynamic evolution, is an important basis for innovation, entrepreneurship, public management, and industrial development in an era of change. The basic elements of the innovation ecosystem are enterprises, universities, and scientific research institutions. Innovation species are the collections of the innovation elements, and the collection of innovation species forms an IP. A variety of IPs are linked together to form various communities in the 
innovation ecosystem. The symbiosis and interaction of IPs contribute to the evolution of the innovation ecosystem. The heterogeneity among various IPs is reflected in the innovation resource input [2], innovation output, and innovation interaction mechanism [3]. How to optimally allocate resources is the basic matter of theoretical research and social practice in the fields of economics [4] and management [5]. How do resource constraints affect relationships between IPs? What is the appropriate interaction mechanism between IPs? These are the main questions in the study that should be answered while constructing an appropriate enterprise innovation ecosystem to effectively improve innovation behavior and competitiveness. If its scale is too small, the IP cannot make full use of the relevant resources in the innovation ecosystem; in this case, innovation resources will be left idle in the innovation ecosystem. If the scale is too large, it will cause the competition of IP for resources in the innovation ecosystem and increase the transaction cost of resource allocation for IP [6].

An appropriate scale and development level of the innovation ecosystem can support the development of IP efficiently. What is the suitable range for the development scale of IP? When the population is on an appropriate scale, the IP will be in a collaborative state, which improves the performance of the IP. In this case, the resources in the innovation ecosystem can be fully utilized, and the innovation performances can be maximized [7]. The impact of industrial clusters on enterprise innovation is considered to be positive in some studies $[8,9]$. However, some literature studies found that the cluster has a negative effect on enterprise innovation $[10,11]$. This study is a development and expansion of organizational ecology. The population scale in a region determines the population density. Existing studies generally use the population density index to analyze the relationship between population scale and innovation performance. Given these two different conclusions, it is necessary to establish a new framework to analyze the relationships between an enterprise's population size and its innovation ability. Therefore, the topic of population ecology should be addressed. Hannan and Freeman [12] analyzed the enterprise problem based on the logic of population ecology, and they founded the organizational ecology field. This present study further develops and expands the population ecology framework.

It is obvious that the research of innovation ecosystem is deepening gradually and that the current research focuses on its concept, characteristics, structure, and operation mechanism. However, few studies are conducted about population dynamics or other ecological methods. The growth characteristics of an innovative ecosystem and its innovative population accord with the laws of ecology, and they need to be verified by relevant ecological theories and methods. Most studies are made from the enterprise level, using ecological models to verify the evolutionary relationship between two subpopulations. In reality, it is more common to form a technological innovation ecosystem where subpopulations are mutually influenced and interdependent. There are few quantitative studies on the relationship between innovation populations.
Population dynamics is often used to analyze the cooperative or competitive relationship between populations. The research shows that the introduction of the population dynamics model into market competition and diffusion produces better analysis results $[13,14]$. The population dynamics model can be adopted to explain the dynamic competition relationship of the stock market [15], product portfolio optimization [16], the symbiotic relationship between competitors in the mobile communication market [17], and the dynamic competition and equilibrium point of TV product population [18]. Based on previous studies, this paper selects interdependent innovation groups as the research object and uses the population dynamics model to study the coordination and balance level between innovation groups in the innovation ecosystem. Different from previous studies, this study, when using the population dynamics model, is not simply based on the scale of innovation groups to explore the collaborative relationship, but based on the number of innovation groups and multiobjective research such as input-output optimization, to explore the appropriate growth scale of innovation groups.

\section{Materials and Methods}

Based on the ecological theory, innovation theory, and population dynamics method, this paper constructs an interaction mechanism model of innovation population. With the objective of resource constraints, population symbiosis effect, and innovation output maximization, the optimal size of the innovation population is determined by a multiobjective programming model. Considering the characteristics of the IP symbiotic system, a comprehensive evaluation method is needed to solve the above multiobjective optimization problem. From the perspective of resource constraints, this paper constructs a dynamic model of population growth in the innovation ecosystem. The suitability of population size is estimated by means of multiobjective programming. This study also constructs a theoretical model from two aspects of input constraints and output maximization, which is a theoretical innovation. Moreover, this research has practical significance, as it provides an appropriate analysis method for each innovation agent to analyze and plan the development scale of the population. The research process of this paper is shown in Figure 1.

As shown in Figure 1, this paper builds population dynamics systems to show the interactive mechanism in the innovation system and builds a multichoice goal programming (MCGP) model for scale optimization.

2.1. Innovation Population Growth Mechanism. According to the logistic model, the internal relationship model of enterprise IP (population 1) is constructed. We can get

$$
g_{1(t)}=\frac{\mathrm{dN}_{1(t)}}{d_{t}}=\alpha_{1} N_{1(t-1)}\left\{1-\frac{N_{1(t-1)}}{K}\right\},
$$

where $g_{1(t)}$ indicates the population growth rate of phase $t$. $N_{1(t)}$ indicates the number of individuals of the population 
in phase $t . N_{1(t)} / K$ represents the number of resources occupied by populations of phase $t$. Within a population of an innovation ecosystem, each unit that occupies resources is defined as $1 / K$.

$$
\begin{aligned}
& \text { Because: } \mathrm{dN}_{1(t)} \approx \Delta N_{1(t)}=N_{1(t)}-N_{1(t-1)}, d_{t} \\
& \approx \Delta t=t-(t-1)=1 \text {, } \\
& \text { So: } \Delta N_{1(t)}=\alpha_{1} N_{1(t-1)}+\beta_{2} N_{1(t-1)}^{2} \text {. }
\end{aligned}
$$

$\Delta N_{1(t)}$ is the number of individuals in a population during the Tperiod. Usually, it is defined as $\alpha_{1}>0$, indicating synergistic effects within populations. $\beta_{2}=-\alpha_{1} / K$, usually $\beta_{2}<0$ representing the internal competition effect of the population. The coefficient of internal competition or population density is called the inhibitory factor.

$$
\begin{aligned}
N_{1(t)} & =\Delta N_{1(t)}+N_{1(t-1)}=\left(\alpha_{1}+1\right) N_{1(t-1)}+\beta_{2} N_{1(t-1)}^{2}, \\
\text { set: } \beta_{1} & =\alpha_{1}+1, \\
\text { get: } N_{1(t)} & =\left\{\beta_{1}+\beta_{2} N_{1(t-1)}\right\} N_{1(t-1)} .
\end{aligned}
$$

If $\left\{\beta_{1}+\beta_{2} N_{1(t-1)}\right\}>1$, then $\Delta N_{1(t)}>0$. The synergistic effect is dominant in the population. Resources within an innovative ecosystem can support an increase in the number of individuals in an IP. Thus, the growth can be sustainable.

If $\left\{\beta_{1}+\beta_{2} N_{1(t-1)}\right\}<1$, then $\Delta N_{1(t)}<0$. The competition effect is dominant in the population. It is difficult to use innovative resources to support the increase in the number of individuals in the IP. Thus, the growth is unsustainable.

Considering the influence of the environment on population, this paper studies the impact of scientific research institution IP on enterprise IP.

$$
N_{1(t)}=\beta_{12} N_{2(t)},
$$

where $N_{2(t)}$ is the number of individuals of scientific research institutions' IP (population 2) in $t$ period and $\beta_{12}$ is the influence coefficient of population 2 on population 1 .

Then, the logistic model can be modified as follows:

$$
g_{1(t)}=\frac{\mathrm{d} N_{1(t)}}{d_{t}}=\alpha_{1} N_{1(t-1)}\left\{1-\frac{N_{1(t-1)}}{K}+\frac{\beta_{12} N_{2(t-1)}}{K}\right\} \text {. }
$$

Equation (4) is substituted by equation (5) to obtain

$$
\Delta N 1(t)^{\prime}=\gamma_{1} N_{2(t-1)}+\gamma_{2} N_{2(t-1)}^{2} .
$$

Among them, $\gamma_{1}=\alpha_{1} \beta_{12}, \gamma_{2}=2 \alpha_{1} \beta_{12}^{2} / K$; we can get

$$
\Delta N_{1(t)}=\left\{\gamma_{1}+\gamma_{2} N_{2(t-1)}\right\} N_{2(t-1)} .
$$

We can judge the relationship between population 1 and population 2 according to the value of $\gamma_{1}$ and $\gamma_{2}$.

If $\left\{\gamma_{1}+\gamma_{2} N_{2(t-1)}\right\}>0$, then $\Delta N_{1(t)}>0$. The population is dominated by the synergy effect, and innovation resources can support the increase of the individual number of IPs, and the growth can be maintained. If $\left\{\gamma_{1}+\gamma_{2} N_{2(t-1)}\right\}<0$, then $\Delta N_{1(t)}<0$. The population is dominated by the competition effect, and it is difficult to use innovation resources to support the increase of the individual number of IPs, and the growth is difficult to maintain.

Considering the influence of the government and various service agencies (such as law firms, accounting firms, consulting companies, advertising companies, and human resources service agencies), formula (4) is further expanded to the following formula:

$$
N_{1(t)}=\beta_{12} N_{2(t)}+\alpha_{\mathrm{zf}} N_{1(t-1)}+\alpha_{\mathrm{fw}} N_{1(t-1)} .
$$

Among them, $\alpha_{\mathrm{zf}}$ is the incentive coefficient of the government to the IP, and $\alpha_{\mathrm{zf}}=j-s . j$ is the preferential policy of the government used to encourage innovation. $s$ is the tax for the impact of various service institutions on the IP.

There is also competition or synergy within the research population. Therefore,

$$
g_{2(t)}=\frac{\mathrm{d} N_{2(t)}}{d_{t}}=\alpha_{2} N_{2(t-1)}\left\{1-\frac{N_{2(t-1)}}{K}\right\},
$$

where $g_{2(t)}$ represents the population growth rate of the T-stage and $N_{2(t)}$ is the number of individuals in the T-stage. $N_{2(t)} / K$ indicates the number of resources occupied by the population in period $T$, and the resources occupied by each unit of a population in the innovation ecosystem are $1 / K$.

Comprehensive consideration of population internal relationship and environmental impact can be shown as

$$
\begin{aligned}
N_{1(t)} & =\left(1+g_{1(t)}+g 1(t)^{\prime}\right) N_{1(t-1)}+\alpha_{\mathrm{zf}} N_{1(t-1)}+\alpha_{\mathrm{fw}} N_{1(t-1)} \\
& =\left\{1+\alpha_{1} N_{1(t-1)}\left(1-\frac{N_{1(t-1)}}{K}\right)+\alpha_{1} N_{1(t-1)}\left(1-\frac{N_{1(t-1)}}{K}+\frac{\beta_{12} N_{2(t-1)}}{K}\right)\right\} N_{1(t-1)}+\alpha_{\mathrm{zf}} N_{1(t-1)}+\alpha_{\mathrm{fw}} N_{1(t-1)} .
\end{aligned}
$$


The following formula can be obtained:

$$
N_{1(t)}=\left(1+\alpha_{\mathrm{zf}}+\alpha_{\mathrm{fw}}\right) N_{1(t-1)}+2 \alpha_{1} N_{1(t-1)}^{2}-\frac{2 \alpha_{1}}{k} N_{1(t-1)}^{3}+\frac{\alpha_{1} \beta_{12}}{k} N_{1(t-1)}^{2} N_{2(t-1)}
$$

Let $1+\alpha_{\mathrm{zf}}+\alpha_{\mathrm{fw}}=\eta_{1}$,

$$
\text { then: } \begin{aligned}
N_{1(t)}= & \eta_{1} N_{1(t-1)}+2 \alpha_{1} N_{1(t-1)}^{2} \\
& -\frac{2 \alpha_{1}}{k} N_{1(t-1)}^{3}+\frac{\alpha_{1} \beta_{12}}{k} N_{1(t-1)}^{2} N_{2(t-1)},
\end{aligned}
$$

because: $N_{2(t)}=\left(1+g_{2(t)}\right) N_{2(t-1)}$

$$
=\left\{1+\alpha_{2} N_{2(t-1)}\left(1-\frac{N_{2(t-1)}}{K}\right)\right\} N_{2(t-1)},
$$

$$
\text { so: } N_{2(t)}=N_{2(t-1)}+\alpha_{2} N_{2(t-1)}^{2}-\frac{\alpha_{2}}{k} N_{2(t-1)}^{3} \text {. }
$$

We can get the partial derivation of the two sides of the equal sign of equations (12) and (14)

$$
\left\{\begin{array}{l}
\frac{\partial N_{1(t)}}{\partial N_{1 t-1}}=\eta_{1}+4 \alpha_{1} N_{1(t-1)}-\frac{6 \alpha_{1}}{k} N_{1(t-1)}^{2}+\frac{2 \alpha_{1} \beta_{12}}{k} N_{1(t-1)} N_{2(t-1)}, \\
\frac{\partial N_{2(t)}}{\partial N_{2(t-1)}}=1+2 \alpha_{2} N_{2(t-1)}-\frac{3 \alpha_{2}}{k} N_{2(t-1)}^{2} .
\end{array}\right.
$$

The second-order partial derivative can be obtained:

$$
\left\{\begin{array}{l}
\frac{\partial^{2} N_{1(t)}}{\partial N_{1(t-1)}^{2}}=4 \alpha_{1}-\frac{12 \alpha_{1}}{k} N_{1(t-1)}+\frac{2 \alpha_{1} \beta_{12}}{k} N_{2(t-1)}, \\
\frac{\partial^{2} N_{2(t)}}{\partial N_{2(t-1)}^{2}}=2 \alpha_{2}-\frac{6 \alpha_{2}}{k} N_{2(t-1)} .
\end{array}\right.
$$

When the population reaches the equilibrium state, the second derivative is zero, and the following equations can be obtained:

$$
\left\{\begin{array}{l}
4 \alpha_{1}-\frac{12 \alpha_{1}}{k} N_{1(t-1)}+\frac{2 \alpha_{1} \beta_{12}}{k} N_{2(t-1)}=0 \\
2 \alpha_{2}-\frac{6 \alpha_{2}}{k} N_{2(t-1)}=0
\end{array}\right.
$$

The only nonnegative solution can be obtained by solving the above equations; that is, the equilibrium point is $\left(k / 3+k \beta_{12} / 18, k / 3\right)$. This equilibrium point represents the equilibrium state of innovation resources occupied by the enterprise IP and scientific research institution IP. By dividing the value of this point by the average amount of innovation resources $(1 / k)$ obtained by each unit of IP, we can get the equilibrium value of the IP scale.
2.2. Multichoice Goal Programming (MCGP). In recent years, multichoice goal programming (MCGP) has been widely used to solve many practical decision-making problems. The multichoice goal programming (MCGP) method proposed by Chang et al. $[19,20]$ is described as follows:

$$
\begin{aligned}
& \text { objective function : } \operatorname{Min} \sum_{i=1}^{n}\left(d_{i}^{+}+d_{i}^{-}\right)+\sum_{i=1}^{n}\left(e_{i}^{+}+e_{i}^{-}\right) \text {, } \\
& f_{i}(x)-d_{i}^{+}+d_{i}^{-}=g_{i}, i=1,2, \ldots, n, \\
& x \in X=\left\{x_{1}, x_{2}, \ldots, x_{m}\right\} \text {, } \\
& g_{i}-e_{i}^{+}+e_{i}^{-}=g_{i, \max }, i=1,2, \ldots, n \text {, } \\
& g_{i, \min } \leq g_{i} \leq g_{i, \max }, i=1,2, \ldots, n \text {, } \\
& d_{i}^{+}+d_{i}^{-}, e_{i}^{+}, e_{i}^{-} \geq 0, i=1,2, \ldots, n, \\
& X \in F, \text { ( } F \text { is the set of feasible solutions). }
\end{aligned}
$$

Here, $d_{i}^{+}$and $d_{i}^{-}$indicate, respectively, the value of the $i$ th goal exceeding and not reaching the expected value of the goal. $f_{i}(x)$ is the objective function of the $i$-th objective. $X$ is the decision variable, representing $m$ alternatives $\left(x_{1}, x_{2}, \ldots, x_{m}\right), g_{i}$ is the expected level for the $i$-th goal.

$e_{i}^{+}$and $e_{i}^{-}$are positive and negative deviation variables close to $\left|g_{i}-g_{i, \max }\right| \cdot g_{i, \text { min }}$ and $g_{i \text {,max }}$ are the lower and upper limits of the target respectively of $g_{i}$. MCGP is a linear form of objective programming, which can be solved by some common linear programming software.

2.3. Materials. China's macroeconomic growth has entered the track of medium-speed development. The primary goal of policy reform is to create a new engine of the economy, cultivate new economic growth points, and finally accelerate industrial transformation and upgrading, and enhance the competitiveness of the real economy by relying on an innovation-driven development strategy. The economic system should not only increase $\mathrm{R} \& \mathrm{D}$ investment and promote investment in the strategic emerging industry but also pay attention to the quality and efficiency of innovation investment. How to make correct innovation investment decisions is very important for different IPs. In China, the government plays a very important role in the innovation ecosystem. The government can adjust the policy according to the growth state of IP under the restriction of resources and promote the development of innovation activities.

Jiangsu Province, a developed province in the east of China and an active area of innovation, is taken as an example. The population quantity equilibrium point of the regional innovation ecosystem is calculated.

Variable interpretation and data selection are as follows: 


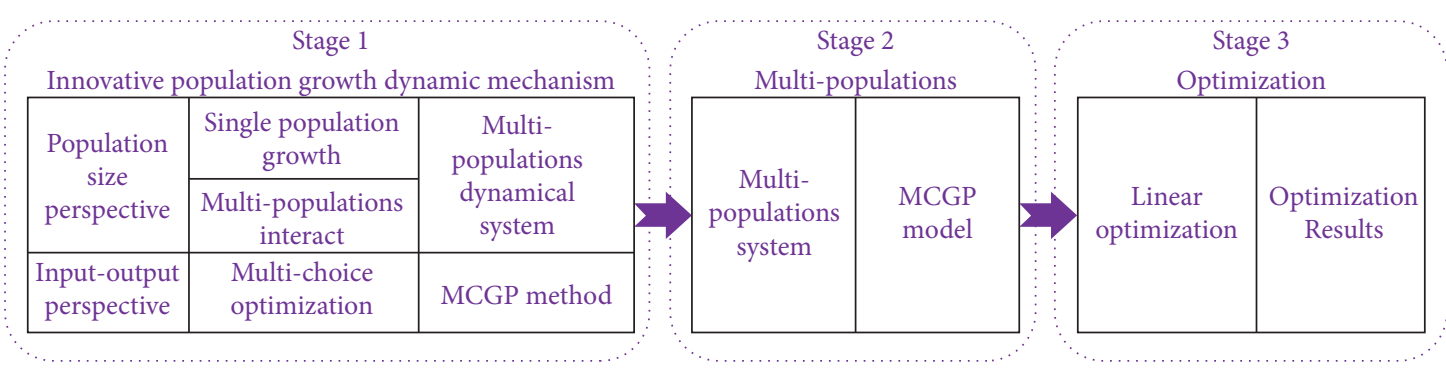

FIgURE 1: Innovation population-scale evaluation process.

(1) $P_{1}$ : population size of innovative enterprises (number of enterprises with scientific and technological activities, unit: number)

(2) $P_{2}$ : population size of scientific research institutions (number of scientific research institutions, unit: number)

(3) $E_{\mathrm{R} \& \mathrm{D}}: \mathrm{R} \& \mathrm{D}$ expenditure (unit: 100 million yuan)

(4) $P_{3}$ : the comprehensive data of scientific research organization $\left(P_{3}=P_{1}+P_{2}\right)$

(5) $\bar{R}$ : the average amount of innovation resources $(\bar{R}=1 / K$, unit: 10,000 yuan $)$

(6) $E_{1}$ : equilibrium solution of equations for population $1\left(E_{1}=k / 3+k \beta_{12} / 18\right.$, unit: 100 million yuan $)$

(7) $E_{2}$ : equilibrium solution of equations for population $2\left(E_{2}=k / 3\right.$, unit: 100 million yuan)

(8) $\mathrm{EP}_{1}$ : equilibrium value of enterprise IP size $(8=6 / 5)$

(9) $\mathrm{EP}_{2}$ : equilibrium value of scientific research institutions $(9=7 / 5)$

The calculated data and the specific data are shown in Table 1. The data in Table 1 are taken from Jiangsu Statistical Yearbook (2001-2018) and relevant calculations are carried out.

As shown in Table 1, from 2000 to 2010 , the actual value of the enterprise IP scale is lower than the equilibrium value, which indicates that, in this stage, innovation resources are relatively sufficient and not fully utilized. From 2011 to 2017, the actual value of the enterprise IP scale is significantly higher than the equilibrium value, and the degree of deviation is increasing. The growth of enterprise IP is constrained by innovation resources.

For the population scale of universities and scientific research institutions, the actual value is higher than the equilibrium value from 2000 to 2003. It shows that the number of universities and scientific research institutions in this period is larger than the equilibrium value. In this period, too many scientific research institutions have occupied the resources of the Jiangsu innovation ecosystem and have squeezed the development space of enterprise IP. From 2004 to 2017, the actual value of IP size of universities and scientific research institutions is lower than the equilibrium value, indicating that, in this stage, innovation resources are relatively sufficient and not fully utilized.
2.4. Empirical Analysis. In this case, the number of authorized patents is taken as a measure of innovation output. The scale of IP suitable for Jiangsu regional innovation ecosystem calculated by Lingo 11 software is shown in Table 2. The objective solution of MCGP and the solution of the resource constraint model (equilibrium value) is used to construct the interval of population suitability (taking the maximum value as the upper limit of the interval and the minimum value as the lower limit of the interval).

Variable interpretation and data selection are as follows:

(1) $\mathrm{MP}_{1}$ : MCGP target solution for population scale of innovative enterprises

(2) $\mathrm{MP}_{2}$ : MCGP target solution for population scale of scientific research institutions

(3) $\mathrm{IP}_{1}$ : suitable interval of scale for population 1 , $\mathrm{IP}_{1}=\left(\mathrm{MIN}\left(\mathrm{MP}_{1}, \mathrm{EP}_{1}\right), \operatorname{MAX}\left(\mathrm{MP}_{1}, \mathrm{EP}_{1}\right)\right)$

(4) $\mathrm{IP}_{2}$ : suitable interval of scale for population 2, $\mathrm{IP}_{2}=\left(\mathrm{MIN}\left(\mathrm{MP}_{2}, \mathrm{EP}_{2}\right), \operatorname{MAX}\left(\mathrm{MP}_{2}, \mathrm{EP}_{2}\right)\right)$

(5) $\mathrm{ARP}_{1}$ : judging whether the scale of population 1 is in the appropriate range (true or false)

(6) ARP2: judging whether the scale of population 2 is in the appropriate range (true or false)

As shown in Table 2, the IP of enterprises in Jiangsu Province is in a suitable range for most of the time, and its scale is appropriate. The population scale of scientific research institutions is out of the appropriate range of the population in most periods. The population of scientific research institutions is not well adapted to the development of enterprise IP and the whole innovation ecosystem. Enterprise IP is the leading IP in the ecosystem, which is at the core of the innovation ecosystem and plays a leading role in the evolution of the innovation ecosystem. Figures can be used to make a more intuitive analysis.

Figure 2 shows that $P_{1}, \mathrm{EP}_{1}$, and $\mathrm{MP}_{1}$ share the same trend, and there is little difference between the three values. The development scale of enterprise IP conforms to the double standard set by the resource constraint model and MCGP model. Enterprises are the main body of the market economy, and the innovation activities of enterprises are more suitable for the market environment. Since 2010, the scale of enterprise IP has increased rapidly. It shows that the innovative ecological environment can promote the rapid 
TABLE 1: Relevant data of empirical analysis.

\begin{tabular}{|c|c|c|c|c|c|c|c|c|c|}
\hline Year & $P_{1}$ & $P_{2}$ & $E_{\mathrm{R} \& \mathrm{D}}$ & $P_{3}$ & $\bar{R}$ & $E_{1}$ & $E_{2}$ & $\mathrm{EP}_{1}$ & $\mathrm{EP}_{2}$ \\
\hline 2017 & 19323 & 1266 & 2260 & 20589 & 1098 & 7699 & 753 & 17409 & 6863 \\
\hline 2016 & 19186 & 1190 & 2027 & 20376 & 995 & 6905 & 676 & 17229 & 6792 \\
\hline 2015 & 18872 & 1113 & 1801 & 19985 & 901 & 6135 & 600 & 16898 & 6662 \\
\hline 2014 & 14150 & 998 & 1653 & 15148 & 1091 & 5631 & 551 & 12808 & 5049 \\
\hline 2013 & 12283 & 944 & 1487 & 13227 & 1124 & 5066 & 496 & 11184 & 4409 \\
\hline 2012 & 11133 & 909 & 1288 & 12042 & 1070 & 4388 & 429 & 10182 & 4014 \\
\hline 2011 & 7712 & 795 & 1072 & 8507 & 1260 & 3652 & 357 & 7193 & 2836 \\
\hline 2010 & 2257 & 714 & 858 & 2971 & 2888 & 2923 & 286 & 2512 & 990 \\
\hline 2009 & 2159 & 713 & 717 & 2872 & 2497 & 2443 & 239 & 2428 & 957 \\
\hline 2008 & 2508 & 693 & 797 & 3201 & 2490 & 2715 & 266 & 2707 & 1067 \\
\hline 2007 & 2236 & 632 & 686 & 2868 & 2392 & 2337 & 229 & 2425 & 956 \\
\hline 2006 & 1831 & 658 & 691 & 2489 & 2776 & 2354 & 230 & 2105 & 830 \\
\hline 2005 & 1695 & 700 & 400 & 2395 & 1670 & 1363 & 133 & 2025 & 798 \\
\hline 2004 & 1569 & 716 & 519 & 2285 & 2271 & 1768 & 173 & 1932 & 762 \\
\hline 2003 & 1271 & 702 & 374 & 1973 & 1896 & 1274 & 125 & 1668 & 658 \\
\hline 2002 & 1222 & 859 & 296 & 2081 & 1422 & 1008 & 99 & 1760 & 694 \\
\hline 2001 & 977 & 744 & 245 & 1721 & 1426 & 836 & 82 & 1455 & 574 \\
\hline 2000 & 968 & 816 & 208 & 1784 & 1166 & 709 & 69 & 1508 & 595 \\
\hline
\end{tabular}

TABLE 2: Solution and scale suitability interval of MCGP model.

\begin{tabular}{ccccccccc}
\hline Year & $\mathrm{MP}_{1}$ & $\mathrm{MP}_{2}$ & $\mathrm{EP}_{1}$ & $\mathrm{EP}_{2}$ & $\mathrm{IP}_{1}$ & $\mathrm{IP}_{2}$ & $\mathrm{ARP}_{1}$ & $\mathrm{ARP}_{2}$ \\
\hline 2017 & 16263 & 1237 & 17409 & 6863 & $(16263,17409)$ & $(1237,6863)$ & $\mathrm{F}$ & $\mathrm{T}$ \\
2016 & 15913 & 1201 & 17229 & 6792 & $(15913,17229)$ & $(1201,6792)$ & $\mathrm{F}$ & $\mathrm{F}$ \\
2015 & 16820 & 1185 & 16898 & 6662 & $(16820,16898)$ & $(1185,6662)$ & $\mathrm{F}$ & $\mathrm{F}$ \\
2014 & 13166 & 1020 & 12808 & 5049 & $(12808,13166)$ & $(1020,5049)$ & $\mathrm{F}$ & $\mathrm{F}$ \\
2013 & 15673 & 886 & 11184 & 4409 & $(11184,15673)$ & $(886,4409)$ & $\mathrm{T}$ \\
2012 & 14206 & 959 & 10182 & 4014 & $(10182,14206)$ & $(959,4014)$ & $\mathrm{T}$ & $\mathrm{T}$ \\
2011 & 9841 & 855 & 7193 & 2836 & $(7193,9841)$ & $(855,2836)$ & $\mathrm{F}$ \\
2010 & 2880 & 768 & 2512 & 990 & $(2512,2880)$ & $(768,990)$ & $\mathrm{F}$ & $\mathrm{F}$ \\
2009 & 2755 & 744 & 2428 & 957 & $(2428,2755)$ & $(744,957)$ & $\mathrm{F}$ \\
2008 & 1816 & 714 & 2707 & 1067 & $(1816,2707)$ & $(714,1067)$ & $\mathrm{T}$ & $\mathrm{F}$ \\
2007 & 1619 & 680 & 2425 & 956 & $(1619,2425)$ & $(680,956)$ & $\mathrm{T}$ \\
2006 & 1326 & 694 & 2105 & 830 & $(1326,2105)$ & $(694,830)$ & $\mathrm{T}$ & $\mathrm{F}$ \\
2005 & 1227 & 681 & 2025 & 798 & $(1227,2025)$ & $(681,798)$ & $\mathrm{F}$ \\
2004 & 1136 & 662 & 1932 & 762 & $(1136,1932)$ & $(662,762)$ & $\mathrm{T}$ \\
2003 & 920 & 655 & 1668 & 658 & $(920,1668)$ & $(655,658)$ & $\mathrm{T}$ \\
2002 & 885 & 794 & 1760 & 694 & $(885,1760)$ & $(694,794)$ & $\mathrm{T}$ \\
2001 & 707 & 687 & 1455 & 574 & $(707,1455)$ & $(574,687)$ & $\mathrm{F}$ \\
2000 & 701 & 754 & 1508 & 595 & $(701,1508)$ & $(595,754)$ & $\mathrm{T}$ \\
\hline
\end{tabular}

growth of enterprise IP. The changing trend of the $\mathrm{MP}_{1}$ variable in 2013 and 2014 is different from that of the other two variables. The main reason is that there are differences in the sensitivity of enterprise innovation population and scientific research institution innovation population to resources. In practice, it can be understood that enterprises are more sensitive to the market environment and their innovation activities are more flexible. Innovation population in 2010 saw a rapid growth rate much higher than the previous growth rate and then maintained a different growth pattern from that before 2010. The main reason for this phenomenon is that the positive impact of innovation investment and industrial policy has increased significantly since 2010.

Figure 3 shows that $P_{2}$ and $\mathrm{MP}_{2}$ share the same trend. There is little difference between the two values. After 2010, $\mathrm{EP}_{2}$ is different from $\mathrm{P}_{2}$ and $\mathrm{MP}_{2}$. The development scale of
IP in scientific research institutions is in line with the standard set by the MCGP model. $\mathrm{EP}_{2}$ has a more similar trend line to $P_{1}$ and $\mathrm{EP}_{1}$. This is the requirement of the coordinated development of heterogeneous populations. However, scientific research institutions cannot adapt to the market environment like enterprises. $P_{2}$ does not make full use of innovative resources to make the population develop faster. This also limits the further development of synergy between $P_{1}$ and $P_{2}$.

2.5. Synergy Evaluation. This paper evaluates the synergy relation with entropy evaluation. Entropy value is always used to measure the chaos in a system. The system is more chaotic while the entropy is larger. When the entropy is smaller, the cooperation will be better $[21,22]$. Let $\langle U, \pi\rangle$ be 


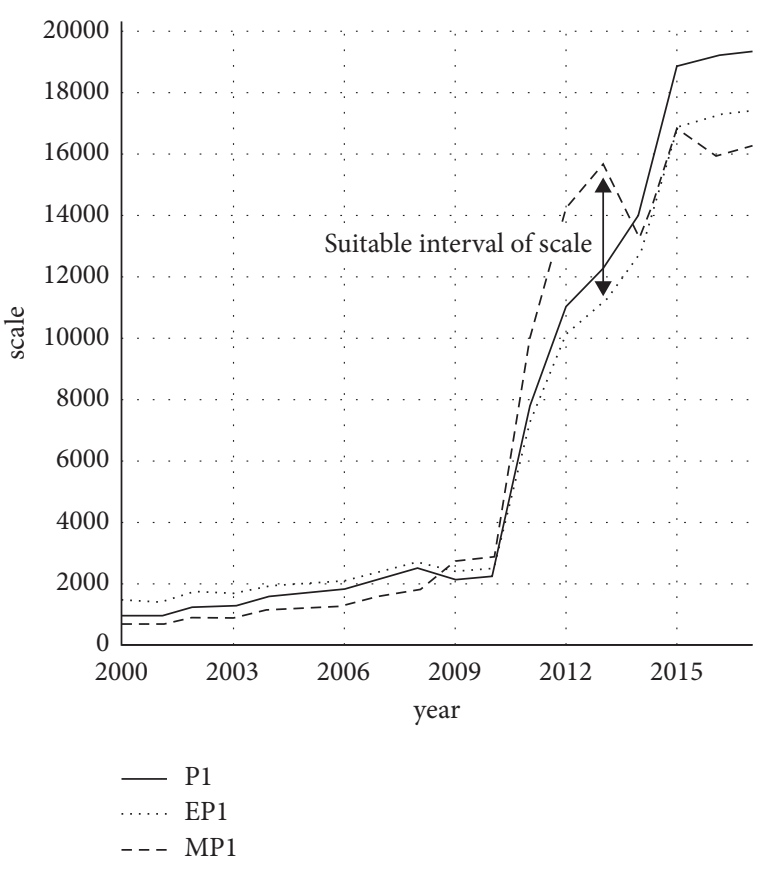

Figure 2: Trend comparison of $P_{1}, \mathrm{EP}_{1}$, and $\mathrm{MP}_{1}$.

an approximation space, where partition $\pi$ consists of blocks $U_{i}, 1 \leq i \leq k$, each of which has cardinality $n_{\mathrm{i}}$. The information entropy $H(\pi)$ of partition $\pi$ is is defined as $[23,24]$

$$
H(\pi)=-\sum_{i=1}^{k} \frac{n_{i}}{n} \log \frac{n_{i}}{n}, \quad n=\sum_{i=1}^{k} n_{i} .
$$

This paper divides entropy into full collaborative entropy and nonfull collaborative entropy. The optimal data entropy of the MCGP model (MP) is full collaborative entropy. The equilibrium solution (EP) and sample data $(P)$ are nonfull collaborative entropy. The nonfull synergy entropy and full synergy entropy are defined as [16]

$$
\begin{gathered}
H_{f}(\pi)=-\sum_{i=1}^{k} \frac{n_{i}}{n} \log \frac{n_{i}}{n}, \quad n=\sum_{i=1}^{k} n_{i}, \\
H_{\mathrm{nf}}(\pi)=-\sum_{i=1}^{k} \frac{n_{i}}{n} \log \frac{n_{i}}{n}, \quad n=\sum_{i=1}^{k} n_{i} .
\end{gathered}
$$
$\left(R_{C}\right)$

This paper gives the definition of collaborative efficiency

$$
R_{C}=1-\frac{H_{f}(\pi)}{H_{f}(\pi)+H_{\mathrm{nf}}(\pi)} .
$$

The full collaborative entropy, nonfull collaborative entropy, and collaborative efficiency of $P, \mathrm{EP}$, and MP are shown in Table 3.

As shown in Table 3, the mean $R_{C}$ of $\mathrm{P}, \mathrm{EP}$, and MP is $0.165,0.205$, and 0.831 . The collaborative efficiency of sample observations is the lowest. The collaborative efficiency of the MCGP model is higher than the collaborative efficiency of the sample observations and equilibrium solution. It can be seen that the system after model optimization has better performance in synergy. This shows that the optimization model in this paper is conducive to the determination of the collaborative scale of the innovation population. The method proposed in this paper is effective.

\section{Results and Discussion}

In this study, the interaction model of IPs is constructed from the perspective of a combination of ecological theory and innovation theory. When the scale suitability of IP in a region is evaluated, it is not comprehensive only from the perspective of resource constraints or output maximization. The resource constraint model based on logistic regression and the multichoice goal programming model are combined to build an analysis path that takes both input and output perspectives into account. At the same time, using the interval value to evaluate suitability makes the population scale suitability evaluation more operable. The results of empirical analysis in Jiangsu Province show that the combination of resource constraint model and multichoice goal programming model can better evaluate the scale suitability of IPs such as enterprises and scientific research institutions in a region. According to the evaluation results, local governments can modify the relevant innovation policies to promote the self-organization evolution of IP.

Interaction relationship within enterprise population is a common topic in the innovation system $[25,26]$. Related researches are held from two perspectives of resource constraints [27] and organizational ecology [28]. It is popular to use the MCGP model to analyze the competition of the innovation population $[16,29]$. In this paper, population dynamics and MCGP are combined to construct a multichoice model. Based on the optimization results, the 


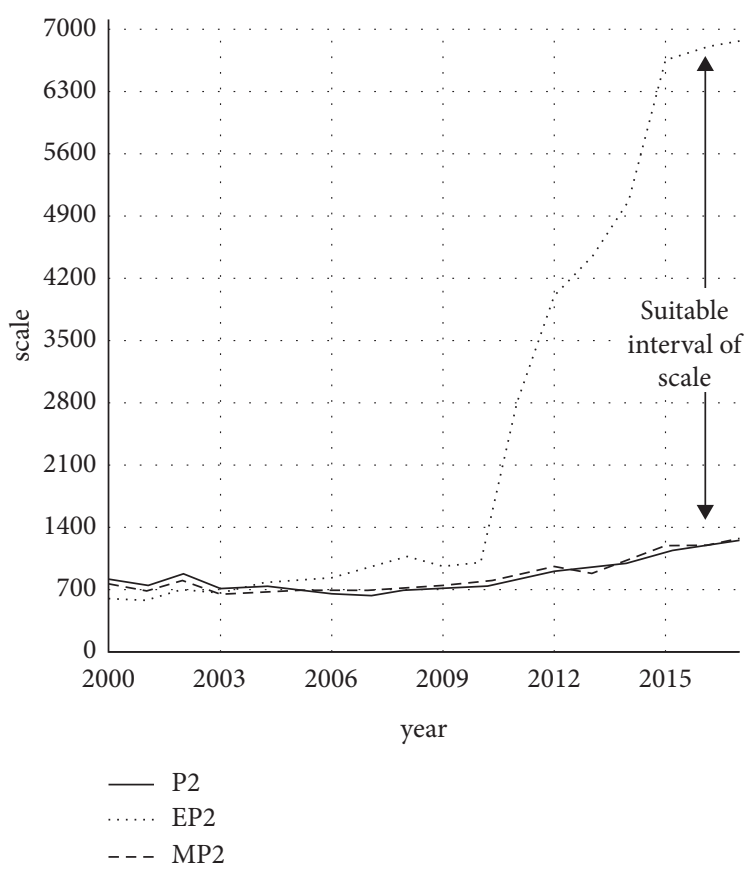

Figure 3: Trend comparison of $P_{2}, \mathrm{EP}_{2}$, and $\mathrm{MP}_{2}$.

TABLE 3: $H_{f}(\pi), H_{\mathrm{nf}}(\pi)$, and $R_{C}$ of $P$, EP, and MP.

\begin{tabular}{|c|c|c|c|c|c|c|c|c|c|}
\hline \multirow{2}{*}{ Year } & \multicolumn{3}{|c|}{$P$} & \multicolumn{3}{|c|}{$\mathrm{EP}$} & \multicolumn{3}{|c|}{$\mathrm{MP}$} \\
\hline & $H_{f}$ & $H_{\mathrm{nf}}$ & $R_{C}$ & $H_{f}$ & $H_{\mathrm{nf}}$ & $R_{C}$ & $H_{f}$ & $H_{\mathrm{nf}}$ & $R_{C}$ \\
\hline 2017 & 1.000 & 0.100 & 0.091 & 1.000 & 0.259 & 0.205 & 0.111 & 1.000 & 0.900 \\
\hline 2016 & 1.000 & 0.097 & 0.088 & 1.000 & 0.259 & 0.205 & 0.110 & 1.000 & 0.901 \\
\hline 2015 & 1.000 & 0.093 & 0.085 & 1.000 & 0.259 & 0.205 & 0.105 & 1.000 & 0.905 \\
\hline 2014 & 1.000 & 0.105 & 0.095 & 1.000 & 0.259 & 0.205 & 0.112 & 1.000 & 0.899 \\
\hline 2013 & 1.000 & 0.112 & 0.100 & 1.000 & 0.259 & 0.205 & 0.091 & 1.000 & 0.917 \\
\hline 2012 & 1.000 & 0.116 & 0.104 & 1.000 & 0.259 & 0.205 & 0.102 & 1.000 & 0.907 \\
\hline 2011 & 1.000 & 0.135 & 0.119 & 1.000 & 0.259 & 0.205 & 0.121 & 1.000 & 0.892 \\
\hline 2010 & 1.000 & 0.239 & 0.193 & 1.000 & 0.259 & 0.205 & 0.224 & 1.000 & 0.817 \\
\hline 2009 & 1.000 & 0.243 & 0.196 & 1.000 & 0.259 & 0.205 & 0.225 & 1.000 & 0.817 \\
\hline 2008 & 1.000 & 0.227 & 0.185 & 1.000 & 0.259 & 0.205 & 0.258 & 1.000 & 0.795 \\
\hline 2007 & 1.000 & 0.229 & 0.186 & 1.000 & 0.259 & 0.205 & 0.264 & 1.000 & 0.791 \\
\hline 2006 & 1.000 & 0.251 & 0.201 & 1.000 & 0.259 & 0.206 & 0.279 & 1.000 & 0.782 \\
\hline 2005 & 1.000 & 0.262 & 0.208 & 1.000 & 0.259 & 0.205 & 0.283 & 1.000 & 0.779 \\
\hline 2004 & 1.000 & 0.270 & 0.213 & 1.000 & 0.259 & 0.206 & 0.286 & 1.000 & 0.778 \\
\hline 2003 & 1.000 & 0.283 & 0.220 & 1.000 & 0.259 & 0.206 & 0.295 & 1.000 & 0.772 \\
\hline 2002 & 1.000 & 0.294 & 0.227 & 1.000 & 0.259 & 0.206 & 0.300 & 1.000 & 0.769 \\
\hline 2001 & 1.000 & 0.297 & 0.229 & 1.000 & 0.259 & 0.206 & 0.301 & 1.000 & 0.769 \\
\hline 2000 & 1.000 & 0.299 & 0.230 & 1.000 & 0.259 & 0.206 & 0.301 & 1.000 & 0.769 \\
\hline Mean & 1.000 & 0.203 & 0.165 & 1.000 & 0.259 & 0.205 & 0.209 & 1.000 & 0.831 \\
\hline
\end{tabular}

population collaborative evaluation is carried out. This method integrates and expands the application fields of the two models, and it is applicable for practical issues.

\section{Conclusion}

The research objective of this paper is to explore a method that can accurately determine the appropriate scale of innovation population development. In order to achieve this goal, this study constructs a dynamic model of the growth of innovative population based on resource constraints model and estimates the suitability of population size with the help of multichoice goal programming method. Two proposed models are constructed to obtain the appropriate population-scale interval. The results show that the research goal of this paper has been well realized.

The theoretical contribution of this paper is that it constructs the theoretical model from two aspects of input constraint and output maximization. The practical significance of the research lies in that it can provide an 
appropriate analysis method for various innovation subjects and government agencies to analyze and plan the development of IP.

The innovation subject should approach the innovation activity with the ecosystem view in the management practice. When making public policies, the government should face up to the objective law of innovation and development. The government should not blindly encourage the scale expansion of enterprises and scientific research institutions; instead, the government should pay attention to the development structure of IP, which easily restricts the inputoutput efficiency of the innovation ecosystem, instead of the scale of IP. Governments at all levels can draw on the ideas of this study and formulate relevant policies to guide the appropriate development of the IP scale. Enterprises and scientific research institutions and other innovative subjects can use the research method of this paper to analyze the innovation competition situation of different industries. Enterprises and scientific research institutions can adjust innovation development strategy and innovation resource allocation based on the results of competitive situation analysis. In this study, the different life cycles of IP development are not considered, and future studies could consider the characteristics of population life cycle development.

\section{Data Availability}

The data used to support the findings of the study are included within the article.

\section{Conflicts of Interest}

The authors declare that they have no conflicts of interest.

\section{Acknowledgments}

This work was supported by the National Social Science Foundation of China (no. 20BGL203).

\section{References}

[1] S. E. Spear and W. Young, "Macroeconomic dynamics survey: endogenous growth theory and models: the "first wave," 1952-1973," Macroeconomic Dynamics, vol. 22, no. 6, pp. 1695-1720, 2017.

[2] C. Beaudry and S. Breschi, "Are firms in clusters really more innovative?" Economics of Innovation and New Technology, vol. 12, no. 4, pp. 325-342, 2003.

[3] E. Giuliani and M. Bell, "The micro-determinants of mesolevel learning and innovation: evidence from a Chilean wine cluster," Research Policy, vol. 34, no. 1, pp. 47-68, 2005.

[4] U. Lichtenthaler, "Open innovation: past research, current debates, and future directions," Academy of Management Perspectives, vol. 25, no. 1, pp. 75-93, 2011.

[5] H. Garriga, G. Von Krogh, and S. Spaeth, "How constraints and knowledge impact open innovation," Strategic Management Journal, vol. 34, no. 9, pp. 1134-1144, 2013.

[6] J. Tan, E. Fischer, R. Mitchell, and P. Phan, "At the center of the action: innovation and technology strategy research in the small business setting," Journal of Small Business Management, vol. 47, no. 3, pp. 233-262, 2009.

[7] R. Pouder and C. H. John, "Hot spots and blind spots: geographical clusters of firms and innovation," Academy of Management Review, vol. 21, no. 4, pp. 1192-1225, 1996.

[8] K. Head, J. Ries, and D. Swenson, "Agglomeration benefits and location choice: evidence from Japanese manufacturing investments in the United States," Journal of International Economics, vol. 38, no. 3-4, pp. 223-247, 1995.

[9] T. Frost and C. Zhou, "The geography of foreign R\&D within a host country - technology selection by multinationals," International Studies of Management \& Organization, vol. 30, no. 2, pp. 10-43, 2000.

[10] J. A. C. Baum and S. J. Mezias, "Localized competition and organizational failure in the manhattan hotel industry, 1898-1990," Administrative Science Quarterly, vol. 37, no. 4, pp. 580-604, 1992.

[11] J. Wang and J. Wang, "An analysis of new-tech agglomeration in beijing: a new industrial district in the making?" Environment and Planning A: Economy and Space, vol. 30, no. 4, pp. 681-701, 1998.

[12] M. T. Hannan and J. Freeman, "The population ecology of organizations," American Journal of Sociology, vol. 82, no. 5, pp. 929-964, 1977.

[13] S. A. Morris and D. Pratt, "Analysis of the lotka-volterra competition equations as a technological substitution model," Technological Forecasting and Social Change, vol. 70, no. 2, pp. 103-133, 2003.

[14] C. Watanabe, R. Kondo, N. Ouchi, and H. Wei, "A substitution orbit model of competitive innovations," Technological Forecasting and Social Change, vol. 71, no. 4, pp. 365-390, 2004.

[15] S.-J. Lee, D.-J. Lee, and H.-S. Oh, “Technological forecasting at the Korean stock market: a dynamic competition analysis using lotka-volterra model," Technological Forecasting and Social Change, vol. 72, no. 8, pp. 1044-1057, 2005.

[16] S.-Y. Wang, W.-M. Chen, and Y. Liu, "Collaborative product portfolio design based on the approach of multi choice goal programming," Mathematical Problems in Engineering, vol. 2021, Article ID 6678533, 16 pages, 2021.

[17] J. Kim, D.-J. Lee, and J. Ahn, “A dynamic competition analysis on the Korean mobile phone market using competitive diffusion model," Computers \& Industrial Engineering, vol. 51, no. 1, pp. 174-182, 2006.

[18] V. B. Kreng and H. T. Wang, "The interaction of the market competition between LCD TV and PDP TV," Computers \& Industrial Engineering, vol. 57, no. 4, pp. 1210-1217, 2009.

[19] C.-T. Chang, "Multi-choice goal programming," Omega, vol. 35, no. 4, pp. 389-396, 2007.

[20] C.-T. Chang, "Revised multi-choice goal programming," Applied Mathematical Modelling, vol. 32, no. 12, pp. 25872595, 2008.

[21] C. E. Shannon, "A mathematical theory of communication," Bell System Technical Journal, vol. 27, no. 3, pp. 379-423, 1948.

[22] I. Düntsch and G. Gediga, "Uncertainty measures of rough set prediction," Artificial Intelligence, vol. 106, no. 1, pp. 109-137, 1998.

[23] M. J. Wierman, "Measuring uncertainty in rough set theory," International Journal of General Systems, vol. 28, no. 4-5, pp. 283-297, 1999.

[24] Y. Y. Yao, "Probabilistic approaches to rough sets," Expert Systems, vol. 20, no. 5, pp. 287-297, 2003. 
[25] R. Cerqueti, F. Tramontana, and M. Ventura, "On the coexistence of innovators and imitators," Technological Forecasting and Social Change, vol. 90, pp. 487-496, 2015.

[26] A. S. Chakrabarti, "Stochastic lotka-volterra equations: a model of lagged diffusion of technology in an interconnected world," Physica A: Statistical Mechanics and its Applications, vol. 442, pp. 214-223, 2016.

[27] S. Baskaran and K. Mehta, "What is innovation anyway? youth perspectives from resource-constrained environments," Technovation, vol. 52-53, pp. 4-17, 2016.

[28] Y. Cui, J. Jiao, and H. Jiao, "Technological innovation in Brazil, Russia, India, China, and South Africa (BRICS): an organizational ecology perspective," Technological Forecasting and Social Change, vol. 107, pp. 28-36, 2016.

[29] -Y. Wang, W.-M. Chen, R. Wang, and X.-L. Wu, "Multiobjective evaluation of co-evolution among innovation populations based on lotka-volterra equilibrium," Discrete Dynamics in Nature and Society, vol. 2021, Article ID 5569108, 14 pages, 2021. 